\title{
Filigrane
}

Écoutes psychothérapiques

\section{La revue Santé mentale au Québec. Syndromes de « la patate chaude " ou du " pas dans ma cour »}

\author{
Aimé Lebeau, Yves Lecomte, Hélène Richard, Dominique Gaucher, Diane \\ Sanésac, Bernard St-Onge et Grégoire Tremblay
}

Volume 18, numéro 1, printemps 2009

Le corps. Sur le divan. Dans le fauteuil I

URI : https://id.erudit.org/iderudit/037728ar

DOI : https://doi.org/10.7202/037728ar

Aller au sommaire du numéro

Éditeur(s)

Revue Santé mentale au Québec

ISSN

1192-1412 (imprimé)

1911-4656 (numérique)

Découvrir la revue

Citer ce document

Lebeau, A., Lecomte, Y., Richard, H., Gaucher, D., Sanésac, D., St-Onge, B. \&

Tremblay, G. (2009). La revue Santé mentale au Québec. Syndromes de " la

patate chaude » ou du « pas dans ma cour ». Filigrane, 18(1), 109-119.

https://doi.org/10.7202/037728ar 


\title{
La revue Santé mentale au Québec. Syndromes de "la patate chaude» ou du "pas dans ma cour»
}

\author{
Aimé Lebeau, sociologue, président \\ Yves Lecomte, sociologue, criminologue, \\ psychologue, directeur \\ Hélène Richard, psychologue, rédactrice \\ de Filigrane \\ Dominique Gaucher, sociologue, \\ Diane Sanésac, psychologue \\ Bernard St-Onge, psychologue, secrétaire- \\ trésorier \\ Grégoire Tremblay, psychologue, \\ Membres du conseil d'administration de \\ Revue Santé mentale au Québec
}

«Les membres du comité ont considéré que la revue Filigrane présentait des articles dont la teneur et la qualité scientifique se trouvent à la limite entre transfert et vulgarisation, et que ceuxci s'adressent davantage à un lectorat de professionnels et du grand public.» (Évaluation du FQRSC, 4 juillet 2008)

«Le comité observe que la revue est de bonne qualité et que les articles sont pertinents dans leur domaine.» (Évaluation du CRSHC, 31 octobre 2008) 
Les deux citations font part aux lecteurs de Filigrane de l'évaluation du FQRSC et du CRSHC de la revue lors du dernier concours de ces deux organismes. Évaluation contradictoire, il va sans dire, mais qui a la caractéristique d'être une répétition de celle du concours de 2005. Comme cette année, le FQRSC avait alors refusé de subventionner Filigrane alors que le CRSHC lui accordait une subvention.

Santé mentale au Québec vit la même situation mais avec la particularité que sa survie est menacée. Pour cette raison, nous lançons une pétition afin de sensibiliser les lecteurs à cette situation. Mais aussi, pour que ses amis en train de la défendre puissent obtenir l'appui nécessaire à leurs actions.

Les lecteurs de Filigrane ne peuvent demeurer indifférents au sort de Santé mentale au Québec. Filigrane est la fille de Santé mentale au Québec. Sans cette dernière, elle n'aurait pas vu le jour.

Dans le respect de cette filiation, nous invitons les lecteurs à appuyer massivement Santé mentale au Québec pour lui assurer un avenir plus radieux que celui qui se dessine.

L n septembre 1976, Santé mentale au Québec publiait son premier numéro dont le sous-titre Vers une nouvelle pratique était annonciateur de l'intérêt actuel du milieu de la santé mentale pour la recherche de pratiques novatrices. Curieux paradoxe, la revue qui a été le porte-flambeau de cette tendance, le lieu de convergence et de diffusion des nouvelles pratiques tout au long des trente-trois dernières années, s'en trouve aujourd'hui évincée. Comment qualifier autrement l'opération de délestage entreprise au cours des dernières années par l'organisme subventionnaire le Fonds québécois de recherche sur la société et la culture (FQRSC) et le ministère de la Santé et des Services sociaux (MSSS) envers la revue ?

Fort heureusement, des appuis se sont manifestés au sein de la Direction de la santé mentale du MSSS pour permettre à la revue de survivre encore le temps d'une «autre année fiscale» et de poursuivre ses activités jusqu'en 2010.

\section{Au-delà de cette date, c'est l'incertitude}

Après avoir publié, entre 1976 et 2008, 67 numéros, environ 15000 pages et 900 articles, et être devenue le dépositaire du patrimoine québécois en santé mentale, SMQ revient à la case départ : être en devoir de justifier son existence, de lutter pour être autre chose qu'un vague souvenir qui enrichit le patrimoine et la devise Je me souviens, et pour ne pas laisser le Québec orphelin d'un outil de diffusion de ses travaux en santé mentale. Autrement dit, il s'agit de lutter pour que le milieu de la santé mentale ne se retrouve pas au point où il en était au cours des années 1960, alors qu'il devait expatrier ses travaux dans les revues étrangères pour les faire connaître. 
Certains qualifient de dépassé ce type de production - et ce qui est encore à nos yeux un projet nécessaire —, qu'on en est à l'heure de la mondialisation, des échanges entre experts dans la lingua franca, l'anglais. Soit ! Publier dans l'anglosphère permet des échanges internationaux, tous en conviennent. Faut-il pour autant annihiler notre spécificité et saboter nos outils culturels? Publier dans la francosphère par l'intermédiaire de Santé mentale au Québec ne permet-il pas aussi des échanges internationaux?

Il y a un lourd prix à payer pour cette mondialisation : se soumettre aux critères des revues internationales, qui reposent sur des valeurs parfois semblables, mais plus souvent différentes de celles du Québec; se plier aux façons de voir du milieu anglophone, en adopter les concepts et les problématiques ; contribuer malgré nous à consolider auprès des chercheurs la prédominance d'une idéologie issue de cette langue et de cette culture. Nous ne pouvons pas accepter une autre résultante de cette domination, à savoir le refus, par une certaine élite du Québec, de reconnaître la valeur d'une revue francophone, et ce, même si, ironiquement ! le milieu international lui accorde suffisamment de valeur pour l'indexer dans Medline! Localisée à Washington, elle est la banque de données internationale par excellence du milieu médical. Depuis 1989, la revue est répertoriée dans cette banque très sélective de données, ce qui requiert une cote minimale de $\mathrm{B}+$, et qui constitue en soi une marque de reconnaissance incontestable. Malgré les tentatives de nombreuses autres revues francophones, seules deux d'entre elles, à notre connaissance, partagent le privilège de Santé mentale au Québec à ce chapitre.

Le prix à payer? S'exposer à la banalisation et à la disparition d'une pensée pourtant ancrée solidement dans les mœurs et les coutumes du Québec. Qu'on le veuille ou non, s'il veut développer son originalité et ses valeurs, un milieu se doit de faire reposer ses outils de diffusion sur une langue de communication spécifique. Autrement, il ne peut qu'emprunter ${ }^{1}$.

C'est grâce au développement de ses outils propres que le Québec s'est fait connaître à l'étranger et que ses expérimentations en santé mentale sont largement citées et servent de référence. Il suffit de rencontrer des collègues de la francophonie européenne pour s'en rendre compte. Doit-on rappeler que, mensuellement, plus de $30 \%$ des 4600 visiteurs du site de SMQ proviennent d'Europe? Quant aux archives de la revue, disponibles sur le site Érudit, elles sont consultées mensuellement par plus de 13000 visiteurs différents, dont une bonne part provient de l'étranger ${ }^{2}$.

\section{La décision de ne plus subventionner la revue ${ }^{3}$}

Que le FQRSC refuse de subventionner la revue depuis 2004, et que le MSSS lui emboîte le pas en 2007 a de quoi surprendre. Un malheureux concours de circonstances, pourrait-on penser. Cette explication tourne court lorsque l'on prend connaissance de l'évaluation de la revue par l'organisme subventionnaire canadien mondialement reconnu: le Conseil de recherches en sciences humaines du Canada (CRSHC).

En octobre 2008, le CRSHC faisait les commentaires suivants : 
«Le comité trouve que, dans l'ensemble, la revue est d'une très grande qualité et que les articles sont pertinents dans leur domaine. Le comité estime que le rédacteur en chef et les membres du comité de rédaction sont bien choisis. Il note favorablement la participation internationale au sein du comité de rédaction. Le comité approuve la clarté et la précision du plan stratégique.»

Il accordait à la revue la note de 87 sur 100 (l'équivalent de la note A), en nette progression en regard de l'évaluation faite sept ans plus tôt ( $80 \%$, soit l'équivalent de la note A-), reconnaissant à la revue une hausse soutenue de ses standards de qualité scientifique et de la pertinence de ses choix éditoriaux.

$\mathrm{Au}$ cours de la même période, le FQRSC faisait de la revue une évaluation inverse, accordant à la revue les notes B + en 2003, puis B en 2007, et refusait, en même temps que le MSSS, de continuer à la financer.

Incongruité dans les évaluations des organismes chargés de faire les évaluations, mais concordance néfaste des décisions de financement... peut-on sérieusement penser que la revue soit la victime d'un malheureux concours de circonstances, comme on pourrait le prétendre? Que le FQRSC et le MSSS refusent presque au même moment de financer la revue ne relève pas du hasard.

Au risque d'analyser de manière un peu clinique des décisions administratives, nous pensons que les deux décisions reposent sur une même logique, symptomatique du syndrome de «la patate chaude» ou du «pas dans ma cour», autrement dit, de l'exclusion. Examinons comment ce syndrome se manifeste au MSSS.

\section{Le MSSS}

Le ministère de la Santé et des Services sociaux soutenait financièrement la revue depuis 1991. Mais, le 22 octobre 2007, la directrice de la Recherche et de l'innovation du MSSS annonçait qu'il retirerait progressivement à la revue le financement qu'il lui avait accordé jusque là, parce qu'il «préconise plutôt l'utilisation des outils spécifiques à cet égard, tel que le programme de soutien aux revues de recherche et de transfert des connaissances du Fonds québécois de la recherche sur la société et la culture (FQRSC, 2007)». À une demande de révision de cette décision adressée au ministre de la Santé et des Services sociaux, la sousministre adjointe répondait, le 20 mai 2008, que le MSSS maintenait sa décision, invoquant qu'il «ne fait pas partie de la mission du MSSS de financer les revues scientifiques ou de vulgarisation scientifique reliées à la recherche».

Se retranchant derrière ce qui nous apparaît être un langage bureaucratique lui permettant de «sauver la face», serait-on tenté de dire, le MSSS renvoie la revue - et la balle - au FQRSC. Or, selon nous, ce programme applique aussi une politique d'exclusion à l'endroit de le revue Santé mentale au Québec.

Revenons à l'affirmation selon laquelle «le MSSS n'a pas pour mission de financer les revues scientifiques ou de vulgarisation scientifique reliées à la recherche». Est-elle fondée? On peut en douter. La revue Développement social 
de l'Institut national de santé publique, pour sa part, reçoit un soutien financier du MSSS qui ne semble pas remis en question. Pourquoi ce «deux poids, deux mesures »? Nous ne pouvons pas nous l'expliquer.

Une rencontre avec le sous-ministre a permis au directeur de la revue de découvrir que cette décision avait été prise à son insu. Le sous-ministre s'est même permis la remarque «Méchante réflexion!» devant le constat qu'il avait fallu 16 ans aux responsables du ministère pour décider que le MSSS n'avait pas pour mission de financer des revues. Heureusement pour la Revue, il s'est montré sensible à l'impact de cette décision sur le milieu de la santé mentale, et il a permis la prolongation du soutien financier du MSSS pour l'année 2009-2010.

Mais une fois encore: au-delà de cette date, c'est l'incertitude.

Comment comprendre une telle décision, alors qu'à son arrivée au pouvoir, le ministre Couillard avait assigné à la santé mentale une place prioritaire ? Comment comprendre que les fonctionnaires n'aient pas jugé essentiel de financer un outil de diffusion des connaissances auprès des intervenants? Comment comprendre qu'on puisse prendre unilatéralement des décisions aussi importantes, sans consulter les principaux intéressés, sans tenir compte de l'impact d'une telle décision sur le milieu de la santé mentale ? Comment expliquer un tel comportement? Fonctionnement en silo, insensibilité à l'impact des décisions sur le milieu concerné, lutte de pouvoir à l'interne ? Serait-ce que la santé mentale ne soit pas, au delà des beaux discours, vraiment prioritaire au MSSS ? Serait-ce que certains responsables du MSSS ne croient plus réellement à la diffusion du savoir, préférant recourir sporadiquement aux experts ${ }^{4}$ ? Même si toutes ces réponses sont plausibles pour l'instant, nous privilégions l'hypothèse suivante: la revue ne cadrerait pas dans la vision globale - et structurante — du système de santé mentale élaborée par les fonctionnaires, dans la longue tradition québécoise de la tabula rasa. Sans doute était-il trop difficile d'imaginer qu'une revue alternative, issue du milieu de l'intervention en 1976, puisse avoir un rôle central dans la diffusion des connaissances au moment où une vague centralisatrice secoue le réseau de la santé ?

Le MSSS ayant préconisé le recours au programme de financement des revues du FQRSC, c'est-à-dire ayant «refilé la patate chaude» au FQRSC si on emploie cette métaphore, examinons la réponse de ce dernier.

\section{Le FQRSC}

Le FQRSC (auparavant le FCAR) finançait la revue depuis le milieu des années quatre-vingt. Vingt ans plus tard, en 2003, il mettait fin à ce financement et ne le renouvellera pas non plus au concours de $2007^{5}$. Les commentaires formulés par l'organisme à l'issue de ce concours - disponibles sur demande auprès de la rédaction de SMQ - soulignent que la qualité, la pertinence et l'originalité des articles, de même que la diffusion de la revue sont «bonnes». Mais l'organisme évalue que la gestion de la revue comporte «des faiblesses importantes».

Laissons le lecteur évaluer si les commentaires émis appuient cette critique sérieuse et la justifient : 
«Sur le plan de la gestion de la revue, les membres du comité d'évaluation soulignent les compétences et le leadership du directeur de la revue ainsi que le souci de la direction, d'une part, de donner accès à la revue à un large public par la plateforme Érudit et dans Medline, et d'autre part, d'assurer la conservation du contenu des versions électroniques. Le comité estime que la politique éditoriale est cohérente avec les buts proposés et que le processus de sélection des articles nous apparaît rigoureux. Le comité de rédaction est diversifié à plusieurs niveaux, notamment parce qu'il est composé de membres de plusieurs disciplines exerçant autant des fonctions de chercheurs universitaires que d'intervenants. Le comité scientifique international apparaît éclectique et regroupe des membres de 32 pays. Toutefois les membres du comité s'étonnent de cette composition puisque la revue n'a d'abonnés que dans six de ces pays et qu'un grand nombre des membres internationaux du comité scientifique ne publie pas dans la revue, et ce, même lorsque la thématique du numéro recoupe la leur. Le comité s'interroge par ailleurs sur les raisons de la parution de deux revues dans une même publication et, par conséquent, aurait apprécié des précisions à cet égard dans la demande.»

Où voit-on, dans ces commentaires, les indicateurs d'une mauvaise gestion? Prenons les éléments un à un. La diffusion à l'étranger? La revue a des abonnés dans neuf pays. Le modèle de présentation des articles? La revue a opté pour une présentation tête bêche afin d'attirer l'attention sur les débats qu'elle publie en sus des dossiers. Le choix des collaborateurs pour une thématique? Le rôle des membres du comité scientifique international est de parrainer la revue, de la diffuser dans leur milieu (auprès des personnes lisant le français pour les pays non francophones), et non d'écrire des articles, même s'ils sont les bienvenus, ni de solliciter des abonnements. Peut-on vraiment, à la lecture des commentaires du FQRSC, parler de «faiblesses importantes»? Au lecteur d'en juger.

Examinons maintenant la gestion directe de la production. Si on omet la participation bénévole des membres des divers comités et des évaluateurs externes, la production et la gestion quotidienne reposent sur l'engagement indéfectible d'un directeur et d'un rédacteur bénévoles. Le premier consacre depuis 35 ans à la revue d'un à trois jours par semaine (selon les époques et les étapes de production des parutions), et le deuxième, environ deux heures par semaine. Une responsable de l'édition consacre à la production trois journées rémunérées par semaine, auxquelles s'ajoutent des heures indispensables... sans rémunération. Une secrétaire et adjointe à la rédaction consacre pour sa part deux jours par semaine à la revue, devant parfois elle aussi y ajouter des heures supplémentaires. L'engagement personnel et la satisfaction de ces deux collaboratrices sont si grands qu'elles sont 
fidèles à la Revue depuis plus de 20 ans, en dépit du fait qu'elles seraient mieux rémunérées ailleurs.

La revue est en équilibre budgétaire depuis 35 ans. En plus de la publication de ses deux numéros annuels, elle a dégagé durant ses premières années un surplus budgétaire qui lui a permis de créer, en 1992, la revue Filigrane, d'orientation psychanalytique, financée d'abord par le Centre national des lettres de Paris, puis, depuis 2004, par le CRSHC.

La corporation à la tête des deux revues a créé un site Internet (http://rsmq. cam.org/) sur lequel on retrouve une documentation papier, audio et vidéo uniques. On a accès aux archives des revues sur deux sites Internet: http://www.teluq. uquebec.ca/smq/et http://www.erudit.org/revue/smq/2008/v33/n2/index. html.

La revue a publié de surcroît huit volumes hors série abordant des problématiques nouvelles qui ne s'intégraient pas dans sa politique éditoriale. Sans porter préjudice aux autres revues financées par le FQRSC, peut-on en dire autant d'elles?

Vu la faiblesse apparente des arguments motivant le refus du FQRSC de financer la revue Santé mentale au Québec, force nous est de supposer que les motifs sont autres.

Le FQRSC fonctionne selon le même modèle catégoriel que le DSM: si une demande ne correspond pas à un certain nombre de critères, elle n'est pas incluse dans le programme. Né de la fusion du FCAR et du CQRS au début des années 2000 , avec pour mandat de se consacrer «au développement du système de recherche et d'innovation québécois en sciences sociales et humaines, en arts et en lettres », le FQRSC soutient financièrement la recherche et la formation des chercheurs, en plus de favoriser la diffusion et le transfert des connaissances, notamment par son programme destiné aux revues.

Or, le FQRSC s'est défini un champ d'action très spécifique susceptible de générer des exclusions hâtives ou mécaniques. Par exemple, si le terme de «schizophrénie » est utilisé dans le titre d'un projet, et même si la recherche en est une qui relève des sciences humaines, le FQRSC refusera de considérer la demande, arguant qu'elle relève du Fonds de recherche en santé du Québec (FRSQ). Notre hypothèse est que la revue Santé mentale au Québec est victime de cette catégorisation. Comme elle traite de santé mentale, elle n'entre pas aisément dans les critères du FQRSC, qui se permet alors d'évaluer la demande de Santé mentale au Québec de manière étroite, à la lumière de son mandat en sciences sociales, et de lui fermer la porte.

Nous en concluons que l'évaluation de la revue faite par le FQRSC n'a rien à voir avec sa qualité. Elle a à voir avec l'étroitesse de l'interprétation de son mandat par le FQRSC, qui refuse d'accommoder une revue ne concordant pas parfaitement avec son orientation de Fonds devant soutenir les sciences humaines et sociales. Mais ne pourrait-on pas, à la rigueur, affirmer que le FQRSC ne respecte pas le mandat, dont il a hérité au moment de son changement de statut, à savoir de financer les revues qui l'étaient par le FCAR? Il ne respecte pas la nature de la 
revue et profite de sa situation pour lui imposer ses critères, comme nous le verrons un peu plus bas. Il veut l'amener à entrer dans son moule.

En ce sens, le FQRSC fait preuve de rigidité et outrepasse, pourrait-on dire, l'esprit du programme des revues. L'intervenant en santé mentale comprendra facilement ce fonctionnement, car il s'apparente aux effets pervers de l'application de la sectorisation en santé mentale, surnommé «le syndrome du code postal». De fait, nous ne sommes pas loin de penser que le comportement du FQRSC à l'égard de la revue Santé mentale au Québec soit symptomatique du début d'un «backlash» du secteur des sciences humaines contre celui de la santé, vu comme un éternel privilégié.

Deux indices ${ }^{6}$ vont dans ce sens. Un premier est apparu dans l'évaluation de 2004. On y lisait ceci : «à la lumière des numéros présentés au concours, le comité perçoit un glissement vers une approche médicale qui n'est pas souhaitable». Un deuxième indice est apparu en 2007: «les membres de comité sont d'avis qu'une plus grande ouverture aux différentes approches et disciplines pour couvrir toutes les dimensions de la santé mentale (par ex. la sociologie ou la criminologie) pourrait augmenter l'impact des articles de la revue, qui leur apparaît limité » (la revue aurait apprécié des précisions à cet égard selon les propres mots du $F Q R S C$ ). Ne peut-on pas voir dans ces propos une pression dirigiste pour éloigner la revue de son orientation multidisciplinaire, et la rendre de plus en plus semblable aux revues de sciences humaines et sociales que finance le FQRC?

La revue nous apparaît donc être la victime de l'approche catégorielle retenue, dans la mesure où ses orientations ne s'y inscrivent pas parfaitement. Depuis sa création, la revue prône une approche multidisciplinaire qui permet l'interaction des principales orientations présentes dans le champ de la santé mentale. Il s'en suit que, dans certains numéros, les thèmes sont plus sociaux; dans d'autres, plus psychologiques, voire carrément médicaux. Parfois, certains numéros abordent des thèmes plus sociologiques ou criminologiques (le numéro d'automne 2009, par exemple, sera consacré à la justice). Mais, que cela plaise ou non au FQRSC, la tendance lourde en santé mentale est médico-psychologico-sociale, et non sociologique ou criminologique, si importante soit-elle. La revue ne peut renier ce fait pour se faire bien voir du FQRSC, et celui-ci ne peut, par ses contraintes, parvenir à changer cette réalité. Le FQRSC doit évaluer la revue en fonction de sa valeur et non pas, comme le laisse penser sa décision, en fonction de catégories ou d'objectifs institutionnels formulés un peu in abstracto. Le procédé choisi actuellement n'a pour effet que de rejeter Santé mentale au Québec, tout comme l'a fait le MSSS.

Pour se dédouaner, le FQRSC ne peut utiliser le prétexte dont s'est servi le MSSS pour refuser de subventionner la revue, puisqu'il n'existe aucun programme de financement des revues au Fonds de recherche en santé du Québec. Il invoque donc d'autres arguments — les seuls, même s'ils sont peu convaincants, qu'il arrive à trouver — comme le lecteur a pu le constater. 


\section{Conclusion}

Les conséquences des décisions du FQRSC et du MSSS relatives au nonrenouvellement du financement de la revue Santé mentale au Québec seront désastreuses pour le milieu de la santé mentale, tant aux plans académiques que cliniques. Faut-il rappeler qu'au cours des 33 dernières années, la revue a été l'outil de références des étudiants, des professeurs et des intervenants, pour leurs travaux, leurs cours et leurs séminaires? Faut-il rappeler que la revue, à de nombreuses occasions, a été à l'avant-garde des orientations du milieu de la santé mentale? Citons, parmi les dossiers importants: La femme québécoise, Vieillir, Où va la psychiatrie? Structures intermédiaires ou alternatives? La réinsertion sociale et, plus récemment, Le rôle des hôpitaux psychiatriques, Le suivi communautaire, Les doubles diagnostics, Les réseaux intégrés de services, etc. Faut-il rappeler que les articles de la revue Santé mentale au Québec servent aussi de référence dans des pays tels la France, la Belgique, la Suisse, la Roumanie, la Tunisie, le Chili et le Sénégal ? Faut-il rappeler, _ et c'est un autre indice de son rayonnement —, le nombre de consultations des archives de la revue sur le site Érudit?

Mais pour bien mesurer l'impact de la disparition de $R S M Q$, il faut poser la question du transfert des connaissances, au cœur des préoccupations actuelles des décideurs et chercheurs. Lisons les propos d'Anne Crocker, psychologue-chercheur, membre de la Commission Kirby et membre du comité de rédaction de la revue:

«Une grand part de l'apport de $R S M Q$ est lié au transfert des connaissances et à la jonction entre recherche et pratique, ce message doit être fort. Il s'agit d'un forum unique francophone qui non seulement apporte de nouvelles pratiques au lectorat mais aussi pose un regard critique sur les tendances, pratiques et recherches en santé mentale. Il s'agit d'une revue ouverte d'esprit capable de tenir compte de multiples perspectives et disciplines (ce qui est maintenant rare dans les tendances de développer des silos de publication). »

Et Emmanuel Stip, psychiatre-chercheur, directeur de la Chaire de schizophrénie de l'Université de Montréal, membre du comité de rédaction de la revue, d'endosser ces propos et de poser la question :

«Même pour les gens du ministère et les praticiens, lisent-ils Science, Nature (deux revues anglophones; NDLR) ou Santé mentale au Québec? Le transfert de connaissances a besoin de relais media et $R S M Q$ est présent, avec en plus une rigueur scientifique. La Société québécoise de schizophrénie lit $R S M Q$ pas Nature.»

Il continue comme suit : 
«La revue est essentielle dans ce champ (Transfer Knowledge) et c'est pourquoi elle est en français. De plus même les Instituts de recherche en santé du Canada reconnaissent le transfert de connaissances comme essentiel: il y aura désormais des fonds de recherche à cet égard: RSMQ est un exemple de cela.»

Pour sa part, Marie-Josée Fleury, chercheur à l'unité de recherche psychosociale de l'hôpital Douglas, éditrice du présent dossier et membre du comité de rédaction de la revue dit ceci :

«La Revue est un véhicule important de diffusion des connaissances pour les praticiens du réseau, véhicule de diffusion des pratiques et modèles d'organisation des soins pour soutenir les changements... véhicule de formation pour les jeunes chercheurs et étudiants qui sont à leur début de publication... (on peut les aider à publier et à écrire...) véhicule pour soutenir la culture d'ici et nos modèles d'organisation de soins...»

Après la lecture de ces propos, peut-on imaginer ce que serait le milieu de la santé mentale depuis 35 ans sans la présence de la revue!

La revue n'a plus à démontrer ni sa pertinence sociale, ni la force de son impact. Il faut être aveugle, ou bien ne pas connaître le champ de la santé mentale pour l'ignorer.

En résumé, les décisions du MSSS et du FQRSC priveront les intervenants, les chercheurs et les décideurs du seul organe francophone de diffusion écrite de leurs travaux. Si ce constat ne suffit pas à convaincre les incrédules, laissons aller le cours des choses.

Heureusement, la Direction de la santé mentale se mobilise en vue d'assurer un avenir à la revue, d'une manière ou d'une autre. Elle assure le financement de la revue pour l'année 2009-2010, de même que le coût des exemplaires distribués gratuitement lors du présent colloque. Elle s'est engagée dans la bataille pour la survie de la revue. Nous la remercions au nom des intervenants du réseau de la santé mentale. Nous remercions aussi les responsables de la TÉLUQ (UQAM) qui ont chaleureusement accueilli la revue lorsqu'ils ont connu ses difficultés financières.

Mais sans réaction vive des intervenants de ce milieu, les fonctionnaires au sein du MSSS et du FQRSC qui sont insensibles aux conséquences de la disparition de la revue ne modifieront pas leur décision. Pour ce faire, il faut manifester votre appui à la Direction de la santé mentale.

Faut-il sauver la revue Santé mentale au Québec ? Certains ont déjà répondu positivement. Nous vous invitons à répondre à votre tour en signant la pétition sur le site Internet de la revue, et en y ajoutant vos commentaires.

http://www.santementaleauquebec.ca/spip.php?rubrique1\&lang=fr 


\section{L'AVENIR DE LA REVUE EST ENTRE LES MAINS DES INTERVENANTS.}

\section{Notes}

1. Nous avons déjà abordé ce sujet dans l'article «Développer de meilleures pratiques», paru dans Santé mentale au Québec au printemps 2003.

2. Les visiteurs proviennent du Canada, des États-Unis, de France, de Belgique, de Suisse, du Maroc, de Côte d'ivoire, d'Algérie, de Tunisie, etc.

3. Les revues québécoises ont accès à deux programmes qui ont pour vocation de financer les revues scientifiques. Le FQRSC qui est l'un d'entre eux, est aussi responsable des subventions destinées aux sciences humaines. Le FQRSC a financé la revue Santé mentale au Québec du milieu des années quatre-vingt à 2003. Le second programme est administré par le Conseil de recherche en sciences humaines du Canada (CRSHC) qui, comme son nom l'indique, administre également un programme de subventions destinées aux sciences humaines. Le CRSHC finance la revue depuis le début des années quatre-vingt-dix. La revue bénéficiait aussi depuis 1991 d'un financement du MSSS.

4. Lire l'analyse de la politique de recours aux experts dans l'article «Évolution de la pensée critique en santé mentale au Québec» paru dans le volume La pensée critique en santé mentale de la revue Santé mentale au Québec.

5. Les numéros évalués en 2007 par le FQRSC et le CRSHC étaient les suivants : La psychothérapie focalisée sur le transfert: Québec-New York et Windigo (printemps 2007); Ethnopsychiatrie (automne 2006); Éthique (printemps 2006); Le soutien social (automne 2005) et Les psychoses réfractaires: modèles de traitement québécois et canadiens (automne 2005).

6. Un autre indice est l'exigence suivante du CRSHC: lors du concours d'octobre 2008, toute recherche présentée dans le volet «santé» devait être accompagnée d'un texte d'une page dans lequel le chercheur expliquait en quoi son projet de recherche respectait les finalités du programme du Conseil. 